\title{
Vascular type 1A angiotensin II receptors regulate renal blood flow and natriuresis
}

Angiotensin II (Ang II)-dependent renal vasoconstriction contributes to blood pressure control, sodium balance and the pathogenesis of hypertension, according to new findings. Matthew Sparks, Thomas Coffman and colleagues suggest that activation of Ang II type 1A $\left(\mathrm{AT}_{1 \mathrm{~A}}\right)$ receptors expressed on vascular smooth muscle cells (VSMCs) results in reduced renal blood flow, leading to increased sodium retention and a rise in blood pressure.

To investigate the vascular actions of Ang II and the potential role of $\mathrm{AT}_{1 \mathrm{~A}}$ receptors in blood pressure control, Sparks, Coffman and colleagues generated mice with specific deletion of $\mathrm{AT}_{1 \mathrm{~A}}$ receptors in VSMCs (SMKO mice). They report that although the mean arterial blood pressure of these mice was significantly lower than that of controls, they showed increased sensitivity to sodium (defined as the change in blood pressure when switched from a low-sodium to a high-sodium diet).
By contrast, the hypertensive response to Ang II infusion was significantly attenuated in SMKO mice compared with controls. The SMKO mice showed an increase in urinary sodium excretion during Ang II infusion, whereas the control mice retained sodium.

\section{$4 \mathrm{AT}_{1 \mathrm{~A}}$ receptors on VSMCs ...} contribute to the development of hypertension by reducing renal blood flow... 77

"Surprisingly, the reductions in blood pressure in SMKO mice occurred despite preserved Ang II-dependent vasoconstriction in the peripheral vasculature, which resulted from compensation by the sympathetic nervous system," say Sparks and Coffman. "However, Ang II-dependent vasoconstriction was largely abolished in the renal circulation, and this effect was associated with a propensity for enhanced sodium excretion."

The researchers conclude that $\mathrm{AT}_{1 \mathrm{~A}}$ receptors on VSMCs directly control Ang II-dependent vasoconstriction in the kidney and contribute to the development of hypertension by reducing renal blood flow and thus increasing sodium retention. "Our research highlights the important contribution of renal vascular function to blood pressure control, sodium handling in the kidney and the pathogenesis of hypertension," comment Sparks and Coffman. "Further exploration of the cross-talk between kidney haemodynamics and sodium excretion could suggest approaches for optimizing hypertension treatment in the clinic."

Ellen F. Carney

Original article Sparks, M. A. et al. Vascular type $1 \mathrm{~A}$ angiotensin II receptors control BP by regulating renal blood flow and urinary sodium excretion. J.Am. Soc. Nephrol. doi:10.1681/ASN.2014080816 